\title{
Semantic memories prime autobiographical memories: General implications and implications for everyday autobiographical remembering
}

\author{
John H. Mace ${ }^{1} \cdot$ Megan L. McQueen $^{1} \cdot$ Kamille E. Hayslett $^{1} \cdot$ Bobbie Jo A. Staley $^{1} \cdot$ Talia J. Welch $^{1}$
}

Published online: 8 October 2018

(C) Psychonomic Society, Inc. 2018

\begin{abstract}
This study investigated the idea that semantic memory activation causes the activation of associated autobiographical memories (e.g., reading the word summer activates knowledge representations in semantic memory, as well as associated personal memories about summer in autobiographical memory). We tested this semantic-autobiographical memory priming hypothesis in three experiments. In Experiment 1, participants were primed with concepts (e.g., summer) on a familiarity task and were then given a word-cue voluntary autobiographical memory task. In support of the hypothesis, the results showed that primed participants had more autobiographical memories overlapping with the primed concepts than control participants. In Experiment 2, participants were similarly primed, but in this case they were given a measure of involuntary autobiographical memory (i.e., Schlagman and Kvavilashvili's (Memory \& Cognition, 36, 920-932, 2008) vigilance task). The results of this experiment also supported the semantic-autobiographical memory-priming hypothesis. Experiment 3 ruled out an alternative possibility (i.e., that autobiographical memory processing had occurred in the word familiarity task) by showing that semantic-autobiographical priming had resulted from a priming task (lexical decision) where autobiographical memory processing was unlikely. The implications of these findings are discussed.
\end{abstract}

Keywords Autobiographical memory $\cdot$ Semantic memory $\cdot$ Semantic priming $\cdot$ Memory models $\cdot$ Episodic memory · Autobiographical memory priming

\section{Introduction}

Throughout the course of a day, one processes massive amounts of information (e.g., reading a newspaper or a book, watching television, engaging in conversation, etc.). Conway has argued that such routine generic information processing continuously activates (or primes) relevant information in the autobiographical knowledge base (e.g., Conway, 2001, 2005). While much of this primed autobiographical knowledge is likely to dissipate with the passage of time, having no influence on cognition or behavior, some of it appears to surface into consciousness immediately as fully formed involuntary autobiographical memories (Mace, 2010b). However, it also seems likely that some primed autobiographical knowledge will influence subsequent autobiographical

John H. Mace

jhmace@eiu.edu

1 Psychology Department, Eastern Illinois University, Charleston, IL 61920, USA remembering, where memories associated with the priming surface as involuntary or voluntary autobiographical memories minutes to hours or perhaps even days later (Mace, 2010b).

In the present study, we examined the possibility that generic processing, in particular semantic activation, can influence (or prime) the content of involuntary and voluntary autobiographical memories. While the impact of such priming may be relatively minimal on the voluntary recall of autobiographical memories in everyday life, we believe that the impact on everyday involuntary autobiographical memories may be substantial, perhaps influencing a great deal of the content of these memories. We review these possibilities below after a review of priming in autobiographical memory.

\section{Priming and activation in autobiographical memory}

Though repetition priming (e.g., where prior exposure to a stimulus speeds its subsequent processing) is likely to occur in autobiographical memory, as it does in other knowledge domains (e.g., the visual word form system, Schacter, 1992), the associative priming model (i.e., where knowledge primes or activates 
related knowledge) has more commonly been invoked (e.g., Conway, 2005; Mace, 2005, 2007a, 2010b) or studied (e.g., Ball \& Hennessey, 2009; Conway, 1990; Mace, 2005; Mace \& Clevinger, 2013) in autobiographical memory (for reviews of associative and repetition priming, see Anderson, 1983a; Roediger \& McDermott, 1993). Theoretically, this model has been used to describe spreading activation between and among various autobiographical knowledge structures (e.g., between lifetime period knowledge and general event knowledge, or among episodic memories - Conway, 2005; Mace, 2005; Mace, Clevinger, \& Bernas, 2013). For the most part, the focus has been on activations and priming within the autobiographical memory knowledge base. However, there has been some focus on priming across knowledge domains (e.g., Conway, 1990), and there seems to be a general consensus that activations within declarative memory are common (e.g., Anderson, 1983a; Barsalou, 1988; Conway, 2001, 2005; Mace, 2010b).

As an example of this, Mace (2010b) argued that activations between semantic and autobiographical memory are commonplace. Evidence for this can be found in studies where semantic categories were shown to prime autobiographical memory (Conway, 1990), where involuntary autobiographical memories have been shown to surface in free-association word tasks (Ball, 2007), and in studies of involuntary autobiographical memories where generic conceptual cues were shown to routinely evoke involuntary autobiographical memories (e.g., Schlagman, Kvavilashvili, \& Schulz, 2007; Ball, Mace, \& Corona, 2007). Mace further argued that the latter set of data provided evidence that a significant part of everyday involuntary autobiographical remembering may be the result of prior generic processing, or semantic-to-autobiographical memory priming.

Priming involuntary autobiographical memories Spontaneous recollection of the past appears to be common in everyday life (e.g., Ball \& Little, 2006; Berntsen, 1996, 1998; Mace, 2004, 2005; Schlagman \& Kvavilashvili, 2008; see reviews in Berntsen, 2009; Mace, 2007b, 2010a). While early studies had placed estimates of the occurrence of involuntary memories to be between three and five per day (Berntsen, 1996, 1998), more recent studies have suggested that their occurrence may be around 20 per day, perhaps outstripping voluntary autobiographical memory occurrence (Rasmussen \& Berntsen, 2011; Rasmussen, Ramsgaard, \& Berntsen, 2015). By definition, involuntary autobiographical memories are random in the sense that one does not intend to remember the past event. However, involuntary autobiographical memory researchers have argued that the content of these memories may not be random, as they may be related to (or primed by) recent processing episodes (e.g., Mace, 2005).

One possibility proposed by Mace (2005) is that involuntary autobiographical memories are primed by routine reminiscence processes (i.e., the act of thinking about or remembering the past). Mace argued that associative priming processes would be at work in such cognitive activities, such that voluntarily retrieved autobiographical memories would activate related autobiographical memories, which may become conscious as spontaneously retrieved memories hours to days later when one encounters cues related to the primed content. To test this idea, Mace had participants come into the lab on multiple occasions where they were asked to recall memories from different lifetime periods (e.g., high school years) in 30-min sessions. These same participants were also simultaneously enrolled in a diary study where they were to record their everyday involuntary memories for a period of 2 weeks. Examinations of the content of these memories revealed there were significantly more memories pertaining to the primed lifetime periods in comparison to the relevant control conditions, thus confirming the fundamental priming hypothesis. Further examinations of the primed memories had also shown that none were repetitions of the memories recalled in the laboratory recall session, thus confirming that the priming processes had fit the associative priming model, and virtually all of the memories had occurred a day (or days) after the priming sessions, thus establishing that the priming effects were long lasting.

More recently, Barzykowski and Niedzwienska (2017) replicated the results of Mace (2005) with a laboratory measure of involuntary memory. In their study, participants were primed in a manner similar to the Mace study (i.e., recalling memories from high school years); however, the effects on involuntary autobiographical memory production were tested with Schlagman and Kvavilashvili's (2008) vigilance task. In this task, participants are presented with a large number of slides containing vertical or horizontal lines with embedded phrases (e.g., drinking from a cup). The task is presented as a study on concentration, where participants are instructed to call out when slides contain vertical lines, and under a separate guise, participants are incidentally instructed to note if they experience involuntary autobiographical memories during the task (see their study for more details, and we explain the vigilance task in greater detail in Experiment 2, below). As in the Mace study, Barzykowski and Niedzwienska found that primed participants produced significantly more involuntary memories from their high school days than unprimed conditions. Barzykowski and Niedzwienska's results also supported the associative priming model by showing that memories were associated, and not repetitions, and that the spread of association extended to areas that had bordered the primed time period (see their study for further details on this result).

Priming voluntary autobiographical memories Unlike involuntary remembering, voluntary recall of the past is a goaldirected process where one knows something about the content of memories before search processes are initiated. It is perhaps for this reason that autobiographical memory 
researchers have not considered how priming may affect the voluntary recall of autobiographical memories. Exceptions to such thinking, however, can be found in Mace (2005) and Mace and Clevinger (2013), where the authors argued that reminiscing about the past may prime and enhance the reminiscence bump in older adults.

The reminiscence bump is a phenomenon where individuals 45 years of age (and older) show a spike in memories from ages 15-25 years (the so-called bump period) in comparison to younger adults, whose memories are more evenly distributed across time periods, displaying a more traditional forgetting curve (e.g., Rubin, Wetzler, \& Nebes, 1986). In Mace and Clevinger (2013), participants were asked to recall memories from different lifetime periods (e.g., elementary school or teenage years) and subsequently given voluntary memory tasks where they were free to recall memories from any period of their life. The results showed that participants were more likely to recall memories from the primed lifetime periods on the second voluntary memory task relative to controls. Among other implications, Mace and Clevinger argued that such autobiographical memory priming could be a factor in the retrieval of bump memories in older adults, as older adults tend to engage in reminiscence behavior (the practice of deliberately recalling memories from one's youth, including the bump period; see Fitzgerald, 1996), and such processes may contribute to the bump by priming memories from this period.

Turning to semantic-to-autobiographical memory priming, while there are a number of studies that can be viewed as evidence that semantic memories prime autobiographical memories generally (e.g., Ball, 2007; Schlagman et al., 2007; see also discussion in Mace, 2010b), Conway (1990) was the first (and only) study to show semantic-toautobiographical priming of voluntary autobiographical memories under certain conditions. In that study, participants in three experiments were primed with taxonomic categories (i.e., category labels such as Birds or Vegetables) or goalderived categories (i.e., category labels such as Birthday Presents or Camping Equipment), and they were then given exemplar cues from each category label (e.g., Sparrow or Potato for taxonomic categories, or Jewelry or Sleeping Bag for goal-derived categories) with the instructions to recall past personal experiences. Priming was then measured as retrieval time on exemplar cues in primed versus unprimed conditions. In all three experiments, goal-derived categories showed shorter retrieval times relative to unprimed conditions, but no priming was evident for the taxonomic category conditions, thus demonstrating semantic-to-autobiographical memory priming in the case of goal-derived categories (see Conway for details on taxonomic and goal-derived categories and their possible relationships with autobiographical memories).

While Conway's (1990) study showed semantic memories can prime autobiographical memories, it is noteworthy that it also failed to find such priming for one class of semantic knowledge (taxonomic categories; see also Conway \& Bekerian, 1987, who also failed to obtain priming in this case). While there may be a number of reasons why these studies failed to find semantic-to-autobiographical priming (we discuss these in the General discussion), one possibility concerns the use of retrieval latencies as the measure of priming. Retrieval latencies may not always be the best measure of priming, particularly when associations between semantic and autobiographical memories might be weak. One possible way to circumvent this potential methodological problem is to use measures that seek to observe activations between semantic and autobiographical memories directly (i.e., that a given semantic prime is seen to activate an autobiographical memory where the content of the two are overlapping).

\section{Semantic-to-autobiographical memory priming: implications for involuntary and voluntary recall of autobiographical memories}

The Mace (2005) study was the first study to establish that autobiographical memories can be primed with other autobiographical memories (i.e., autobiographical-to-autobiographical memory priming; Mace, 2010b), and that such priming is associative in a manner similar to priming observed in semantic memory, where memories will activate other associated memories. The study was also the first to suggest that routine cognitive processes such as reminiscing were capable of priming everyday involuntary autobiographical memories, potentially accounting for a significant proportion of everyday involuntary autobiographical memory production.

In the current study, we propose that generic processes will cause similar associative priming processes to occur with involuntary autobiographical memories. For example, reading or hearing the word Garden or School will activate a pool of autobiographical memories associated with these concepts, and, as in the case of the reminiscence priming discussed above, some of these memories will now be capable of surfacing as involuntary autobiographical memories some time later (e.g., minutes to hours) when processing contexts overlap with the primed content. If such a model of semantic-toautobiographical memory priming is true, then this could potentially account for a significant amount of daily involuntary remembering, given the massive amount of such processing that occurs in the course of a day.

Regarding voluntary remembering, we further propose that generic processes will affect the voluntary recall of autobiographical memories, and that such priming may routinely affect voluntary remembering in everyday life. We believe there are a number of ways in which this can happen. One possibility, which appears to be borne out by Conway's (1990) data, is that semantic priming may facilitate retrieval when the primed content overlaps with the to-be-remembered autobiographical information (i.e., either by making it more likely to be retrieved 
or making retrieval more fluent). On the flip side, though possibly a rare occurrence, there might be scenarios where semantic priming functions to have negative effects on voluntary recall (e.g., somehow the primed content blocks or inhibits the retrieval of a target autobiographical memory). Another possibility is that prior semantic activations could affect the content of memories when one is engaged in reminiscing about the past in a somewhat non-specific manner; for example, freely recalling a particular lifetime period, such as when I was a teenager or lived in New York City. This argument is similar to the one proposed by Mace and Clevinger (2013); however, in this case it is the proposition that voluntary remembering of the past in general may be influenced by prior semantic processing. (We focused on this last possibility in the current study.) If any (or all) of these possibilities are true, it would indicate that voluntary recall, a process that is considered intentional and non-random, can be random when prior content, unbeknownst to the individual, influences the selection of memories.

\section{Overview of study}

In this study, we tested the aforementioned associative priming hypothesis, which states that semantic activations cause activations of associated autobiographical memories (e.g., reading the word garden activates a pool of personal memories surrounding this concept), and this semantic-toautobiographical priming process subsequently influences the content of voluntarily and involuntarily retrieved autobiographical memories (e.g., in reminiscing about one's childhood, you remember how you used to help your grandmother in her garden, or hearing someone say that they were gardening spontaneously triggers the same memory). We reasoned that evidence in support of this hypothesis would serve three general purposes. One, it would support the general notion that priming occurs on a widespread basis in declarative memory, adding to a very small body of research that demonstrated semantic-to-autobiographical priming (e.g., Conway, 1990). Two, it would support the idea that everyday voluntary recall of the past is affected by such semantic-toautobiographical priming. Three, it would support the idea that the content of everyday involuntary autobiographical remembering is influenced by semantic-to-autobiographical priming.

We tested the semantic-autobiographical memory priming hypothesis in three experiments. In Experiment 1, participants were primed with common concepts (e.g., music, summer, car), after which they were given a voluntary autobiographical memory task, where it was hypothesized that the content of autobiographical memories would overlap with the primed semantic content. Though previous work has examined semantic-autobiographical priming in voluntary autobiographical memory tasks (Conway \& Bekerian, 1987; Conway, 1990), these studies only assessed the influence of semantic primes on autobiographical memory retrieval speed, not on the content remembered in the autobiographical memory task. We believe that content priming is a stronger measure of semantic-autobiographical memory priming than retrieval latencies. Experiment 2 employed the same semantic priming method; however, in this case we assessed the effects of semantic priming on content in an involuntary autobiographical memory task (Schlagman \& Kvavilashvili, 2008). Experiment 3 was conducted to rule out a potential confound with the priming tasks used in Experiments 1 and 2.

\section{Experiment 1}

In Experiment 1, we activated semantic memories with a word familiarity task. Here, participants were given a list of words (e.g., music, summer, quip) for which they were to rate the familiarity of word meanings on a scale of $0-5$. Following the word familiarity task they were given a word-cue autobiographical memory task, whose cues (e.g., flower) did not overlap with words on the word familiarity task. A second group of participants (deemed the control group) were engaged solely in the autobiographical memory task.

Word familiarity tasks have been used frequently in language studies (e.g., Connine, Mullennix, Shernoff, \& Yelen, 1990), and we selected this task because we envisaged it as a relatively powerful means of activating concepts in semantic memory. The rationale for using different cues on the two tasks was straightforward. Given that we wanted to observe content priming directly rather than indirectly with retrieval latencies, as in Conway (1990), we had to use different cues across tasks because matching cues (i.e., where the prime is music and the retrieval cue is music) would require one to use retrieval latencies as the priming measure, as participants in both the priming and control groups are instructed to recall memories in response to the retrieval cues. Also, in using different word cues on the autobiographical memory task, we hoped to simulate the type of free-recall reminiscence that might be affected by semantic-autobiographical priming in everyday life. For example, in reminiscing about a past lifetime period (e.g., when one lived in New York City), one is free to select from a set of different memories associated with the time period. Similarly, in a word-cue autobiographical memory task, one is free to select from a range of memories associated with given cues (e.g., memories associated with flower). Thus, we reasoned that showing that the word cue flower, for example, had caused a memory about music to be retrieved should be powerful evidence for this proposition, as this would be analogous to remembering that one used to listen to music in Central Park after having been primed with the concept earlier in the above reminiscence scenario.

Concerning predictions, we predicted that the priming group would show more autobiographical memories associated with 
concepts on the word familiarity task than the control group, and such observations can be taken as good evidence that the activation of concepts on the word-familiarity task had primed associated autobiographical memories.

\section{Method}

\section{Participants}

The participants were 43 undergraduate students from Eastern Illinois University who participated in exchange for course credit. Twenty-two of the participants served in the priming group, and 21 in the control group. Thirty-one of the participants were females (15 in the priming group, 16 in the control group), and the age range of the participants was from 18 to 21 years $(M=19.1$ years $)$.

\section{Materials and procedure}

The word familiarity task consisted of 34 relatively high (e.g., music) and low (e.g., quip) frequency words (see Appendix 1), printed and presented in booklet form. The high-frequency words had frequency counts in the Corpus of Contemporary American English ranging from 6,149 to 356,731 ( $\mathrm{m}=$ 81,836 ), while the low-frequency words had a range of $77-$ $679(m=385)$. We should note that we were not interested in manipulating word frequency, but instead, there were different reasons why some of the items were high-frequency words, and some low-frequency words. The high-frequency words were selected for their likelihood of being associated with personal experiences (e.g., summer). We selected them from participant protocols collected in our lab in connection with numerous past studies on autobiographical memory (i.e., some published studies - Mace, 2005, 2006, and some unpublished). Some of the items appeared on numerous occasions as topics in personal memories (e.g., school, friends), some occurred at intermediate levels (e.g., pet, music), and some on rare occasion (e.g., doctor, art). This selection process helped to ensure that our items were likely to be associated with personal experience, and therefore capable of being primed. As it turned out, these items were high-frequency words. The low-frequency words were selected simply to legitimize the cover story of the task by creating a range of familiar and unfamiliar concepts. It should also be noted that we did not expect that any of the low-frequency words would prime autobiographical memories; given their very low degree of familiarity and usage as words (e.g., quip, garrulous), none had appeared in any of the above-mentioned protocols.

The autobiographical memory task consisted of 15 word cues (e.g., flower, see Appendix 2), printed and presented in booklet form. The list was used in other voluntary autobiographical memory tasks used in our lab (e.g., Mace \& Clevinger, 2013). None of the words used on the autobiographical memory task were repetitions of words used in the word familiarity task, nor were they synonyms, or words that might be perceived as obvious associates of these words. We determined associative status by casual inspection, as we were only looking to eliminate strong (or obvious) associations. As stated at the outset, the reason for non-overlap in the cues was so we could directly observe content priming in circumstances that were similar to everyday reminiscing.

All participants were tested individually. Priming group participants were first engaged in the word familiarity task, followed by the autobiographical memory task. Each task was presented to participants as a separate study. The time lag between the priming task and the memory task was approximately $5 \mathrm{~min}$ on average. Control group participants were only engaged in the autobiographical memory task. In the word familiarity task, participants were told they were being involved in a study on familiarity with word meanings (i.e., definitions). They were instructed to consider the definitional meanings of words, and then rate them using a $0-5$ scale, where 0 indicated word definitions were unknown, 1 indicated word definitions were very unfamiliar, and 5 indicated high familiarity with word meanings. The ratings were recorded in the word familiarity task booklet, entered to the right of each word. In the autobiographical memory task, participants were instructed to use the word cues to recall specific personal experiences from their past (i.e., episodic memories) that were related to the word cues. Participants entered their memories in the autobiographical memory task booklet, recording them underneath the word cues. Both the word familiarly and the autobiographical memory tasks were self-paced. In cases where participants were unable to recall memories to specific cues on the autobiographical-memory task, the participant decided when to move onto the next word cue. Following autobiographicalmemory task, both groups were instructed on the differences between general (i.e., more abstract autobiographical memories, such as "I went to London in 2005") and specific autobiographical memories (i.e., episodic memories, such as remembering "having a flat tire while driving across the Tower Bridge"), and they were asked to read through their memories, marking which were general and which were specific.

\section{Categorization method}

The content of the autobiographical memories for both the priming and the control groups was read by two independent judges. The judges were looking for memories that clearly were personal memories involving the concepts presented on the word familiarity task. For example, personal memories involving, car, music, summer, hiking, and so forth (e.g., I remember listening to music in the park, or I remember hiking in the mountains). Memories were deemed as primed (or conceptually overlapping) memories only in cases where the content of the memory clearly involved the content of items from 
the priming list, as in the examples given. Memories were deemed primed (or overlapping) if they involved the explicit (e.g., I remember when I was listening to music with ....) or implicit (e.g., I remember when I was listening to Beethoven's 5th with ....) description of a concept, as conceptual overlap was the critical factor. In no event were memories deemed primed memories if words were used to simply describe aspects of an event (e.g., I remember the teacher quipped .....), eliminating possible instances of repetition priming, or if they were used as tangential references (e.g., I remember we spent 3 weeks in Florida, visiting Disney Land, ..., and I think we traveled by car on that trip). In cases where memories contained overlapping content from two (or more) of the word-familiarity items, the memories were only counted once in the scoring process. Any disagreements between the judges were settled through discussion.

\section{Results and discussion}

An examination of the familiarity ratings in the priming group showed that the overall ratings were $3.73(S D=0.24)$, while a breakdown of these ratings showed they were $4.90(S D=0.13)$ for high-frequency words, and $0.97(S D=0.61)$ for lowfrequency words. Regarding the memory measures, we first analyzed performance on the word-cue task in terms of overall memory production, and general/specific memory production. This analysis showed participants had very high rates of autobiographical memory generation on the word-cue task (95\%, priming group, $97 \%$, control group). Of these memories, 70 $72 \%$ were specific autobiographical memories (70\%, priming group, $72 \%$, control group, $\left.\chi^{2}(1)<1.0\right)$, with the balances being general or summary autobiographical memories.

Turning to the main question of interest, an analysis of overlapping content between items on the word familiarity task and the autobiographical memory task showed that .30 ( $S D=.11)$ of the priming group's memories were personal memories concerning the concepts on the word familiarity task (e.g., memories about summer or music), whereas the proportion was .19 $(S D=.09)$ for the control group, representing baseline probability (the Kappa statistic indicated high concordance between the judges, $K=.88, S E=.05$ ). We subjected these data to an independent-samples $t$-test, which showed the proportional overlap was statistically higher in the priming group, $t(41)=3.47, p=.001, d=1.09$, thus indicating support for the semantic-autobiographical priming hypothesis.

A further analysis of specific/general memories production rates in the conceptually overlapping data showed specific memory rates similar to the overall data reported above (77\%, priming group, $74 \%$, control group, $\left.\chi^{2}(1)<1.0\right)$. An additional examination also showed that all of the primed memories had corresponded to the high-frequency words contained on the word-familiarity task, with none of the low-frequency items being represented in either group (18 of the 23 high-frequency items were represented in the priming group, $78 \%$, with many of the items occurring frequently as memory topics; 14 of the 23 in the control group (61\%) occurring with less frequency as memory topics overall).

Finally, at debriefing, participants indicated they had no knowledge of the research hypothesis, thus ruling out the possibility that the results were due to deliberate recall of memories associated with the items on the word-familiarity task.

In sum, showing that the priming group had more memories overlapping with items on the word familiarity task than the control group supported the semantic-autobiographical priming hypothesis. The results were consistent with the notion that the activation of semantic memories on the word familiarity task had led to the activation of personal memories associated with the concepts, and consequently, some of these memories surfaced on the autobiographical memory task. We believe that these findings offer powerful support for the semanticautobiographical priming hypothesis because these activations occurred in a cueing environment that was very dissimilar to the word familiarity task items (e.g., one recalls a memory involving music, when the word cue flower). We believe these findings suggest that the semantic-autobiographical priming in everyday cognition could influence the content of memories recalled when one is reminiscing about the past. We return to this point in the General discussion.

\section{Experiment 2}

In Experiment 2, we examined the influence of semanticautobiographical priming on involuntary autobiographical memories. To accomplish this we used the Schlagman and Kvavilashvili (2008) method for measuring involuntary autobiographical memories in the laboratory. In this approach, participants are presented with a large number of slides containing either vertical or horizontal lines. Embedded within each slide is a simple phrase (e.g., drinking from a cup), which participants are told to ignore. Participants are also told that they might experience spontaneous thoughts or memories during the task, and if so, they are to note them. This method has been shown to reliably elicit involuntary autobiographical memories in a number of studies beyond Schlagman and Kvavilashvili's initial report (e.g., Barzykowski \& Niedzwienska, 2017; Barzykowski \& Staugaard, 2016; Vannucci, Pelagatti, Hanczakowski, Mazzoni, \& Paccani, 2015).

We used a slightly modified version of the Schlagman and Kvavilashvili (2008) method in the current experiment. Priming group participants received the same wordfamiliarity task used in Experiment 1 and subsequently they were engaged in the Schlagman and Kvavilashvili involuntary memory task (hereafter known as the vigilance task), while control group participants only received the latter task. Consistent with the semantic-autobiographical priming 
hypothesis and the results of Experiment 1, we predicted that the priming group would report more involuntary autobiographical memories concerning the concepts on the wordfamiliarity task than the control group.

\section{Method}

\section{Participants}

The participants were 40 undergraduate students from Eastern Illinois University who participated in exchange for course credit. Twenty of the participants served in the priming group, and 20 in the control group. Thirty-two participants were females (16 in each group), and the age range of the participants was from 18 to 25 years $(M=19.7$ years).

\section{Materials and procedure}

The word familiarity task was the same as in Experiment 1. Stimuli for the vigilance task consisted of 512 slides containing either horizontal or vertical lines, each with a unique word phrase (e.g., going to work) embedded in the center of the slide. 477 of the slides contained horizontal lines, 35 contained vertical lines. The slides were presented randomly for $1.5 \mathrm{~s}$ on a computer screen, via the SuperLab (version 4.5) software, for a total duration of approximately $13 \mathrm{~min}$. Given the commonality of some of the words used in the word familiarity task (e.g., friend or pet), some of the embedded phrases contained those words (14 in total, with a couple appearing twice, e.g., making a new friend; missing a friend), some could be seen as associated with the concepts used in the word familiarity task (38 in total, e.g., getting $a \mathbf{d o g}$ ), while most were unrelated (460 in total, i.e., having no apparent relation to word familiarity items, e.g., going to work).

The design of the vigilance task matched Schlagman and Kvavilashvili's (2008) with some minor, insignificant, variations. For example, we used 512 slides instead of the $600-800$; 35 , instead of 11-15, contained vertical lines; and the slides were presented randomly rather than in a fixed order. A more substantial variation included instructions on task-unrelated thoughts. In the current study, we asked participants to report both task-unrelated thoughts and involuntary memories (see below), while Schlagman and Kvavilashvili asked participants to only report involuntary memories. Concerning the phrases embedded within the slides, there are two reasons why we were not concerned that some of them matched (or were associated with) items from the word familiarity task. One, given that the vigilance task is an involuntary memory measure, as opposed to a voluntary memory measure, priming is therefore measured by the presence (or absence) of spontaneous memories across conditions. Thus, comparisons can be made across primed and unprimed conditions regardless of cue type (i.e., related or unrelated cues). Two, in Experiment 1, we were interested in creating conditions that were analogous to everyday reminiscing, which was best done with non-matching cues. We did not have that same need (or interest) in the current experiment, as reminiscing is a voluntary memory process, and we were only interested in involuntary memory processes here.

Concerning the procedure, all participants were tested individually. Priming group participants were first engaged in the word familiarity task, followed by the vigilance task. Each task was presented to participants as a separate study. The time lag between the priming task and the memory task was approximately $5 \mathrm{~min}$. The control group participants were only engaged in the vigilance task. The instructions for the word familiarity task were the same as in Experiment 1 . In the vigilance task, participants were told that they were being involved in a study on concentration. They were told they would see slides with either horizontal or vertical lines, and they were to say "yes" out loud whenever the slides contained vertical lines. They were further told that the slides would also contain phrases, but they were to ignore them. To ensure their understanding of the instructions, they then received four practice slides, one containing vertical lines. Once it was clear that they understood, they were further instructed that it was possible that they may experience task-unrelated spontaneous thoughts or memories (the concept of involuntary memories was explained to them), and if they experienced one or the other, they were to click the mouse and record them in a booklet that contained sheets of lined paper. Once this aspect was understood, the task commenced. On trials where participants clicked the mouse, an instruction screen would pop up reminding them to record their thoughts or memories in the booklet, clicking the mouse again when finished to return to the vigilance program. Following the vigilance task, both groups were instructed on the differences between general and specific autobiographical memories, and they were asked to read through their booklet, marking entries as either spontaneous thoughts or memories, noting general or specific in the latter case.

\section{Categorization method}

Using the same criteria specified for Experiment 1, the judges examined the memories of the priming and the control group.

\section{Results and discussion}

An examination of the familiarity ratings in the priming group showed that the overall ratings were $3.74(S D=0.25)$, while a breakdown of these ratings showed they were $4.91(S D=$ $0.14)$ for high-frequency words, and $0.99(S D=0.60)$ for low-frequency words. Concerning the overall report of memories or thoughts on the vigilance task, the priming group reported an average of $8.65(S D=4.02)$ memories and 1.60 $(S D=1.69)$ thoughts on the vigilance task, whereas the control group reported an average of $7.75(S D=2.79)$ memories 
and $1.50(S D=2.26)$ thoughts. Two participants reported no memories (one in priming and one in control). A series of ttests found no significance between the groups in either the memories or the thoughts reported $(t \mathrm{~s}<1.0)$. Of the memories reported, $81-82 \%$ were specific memories $(81 \%$, control group; $82 \%$, priming group, $\left.\chi^{2}(1)<1.0\right)$.

Turning to the main question of interest, an analysis of the memory content showed that $.49(S D=.21)$ of the priming group's memories were personal memories corresponding to items on the word familiarity task, whereas the proportion was $.29(S D=.21)$ for the control group, representing baseline probability (the Kappa statistic indicated high concordance between the judges, $K=.86, S E=.06$ ). We subjected these data to an independent-samples $t$-test, which showed the proportional overlap was statistically higher in the priming group, $t(36)=$ $3.03, p<.01, d=0.98$, thus indicating significant semanticautobiographical priming. A further analysis of the overlapping memories showed that $17 \%$ of those in the priming group were triggered by cues that either contained items from the familiarity task (e.g., making a new friend) or were related to them (e.g., getting a dog), while the rate was $7 \%$ in the control group, thus indicating that most of the primed memories were triggered by unrelated cues (i.e., phrases not containing the same or related items). Statistical analyses conducted on the related (i.e., having the same or related items) and unrelated set of cues showed that the priming group generated more overlapping memories than the control group in the case of related $\left(\chi^{2}(1)\right.$ $=6.25, p=.01)$ and unrelated cues $\left(\chi^{2}(1)=5.24, p=.02\right)$, thus indicating that priming occurred in both of these cue categories.

Additionally, as in Experiment 1, the primed memories only corresponded to the high-frequency words on the familiarity task, with 18 of the 23 high-frequency items being represented in the priming group (78\%), with many items occurring frequently as topics; 15 of the 23 in the control group (65\%), occurring with less frequency overall. Concerning specific memory production rates in the primed memories, they were similar to the rates observed in the overall memory data reported above $\left(80 \%\right.$, priming group; $83 \%$, control group, $\left.\chi^{2}(1)<1.0\right)$.

Finally, at debriefing, participants indicated they had no knowledge of the research hypothesis or the underlying purpose of the vigilance task, thus ruling out the possibility that the results were due to deliberate recall of memories associated with the items on the word-familiarity task or deliberate recall in general.

In summary, the priming group showed more memories conceptually overlapping with items on the word familiarity task than the control group. As in Experiment 1, this result supports the general notion that semantic memories prime autobiographical memories. However, as we have now obtained the result with involuntary autobiographical memories, this finding has a different set of implications for this class of memories. In particular, we believe that this result suggests that at least some of the content of everyday involuntary autobiographical memories may be influenced by semanticautobiographical memory priming. We return to this point in the General discussion.

\section{Experiment 3}

It could be argued that priming from the word familiarity task was a function of autobiographical-to-autobiographical memory activations rather than to semantic-to-autobiographical memory activations. That is, in order to make familiarity judgements about concepts, participants may have retrieved their own personal experiences with the concepts, and therefore, these autobiographical memories were responsible for the priming observed in subsequent memory measures (i.e., autobiographical-to-autobiographical memory priming). To rule out this possibility in the current experiment, we used lexical decision as our priming task. In lexical decision, participants simply decide whether items are words or not. Given the low cognitive demand of a lexical decision task, it seems highly unlikely that participants would need to retrieve their personal experiences with words in order to judge their lexical status.

Thus, in the current experiment, participants received a lexical decision task and priming was subsequently assessed with a voluntary autobiographical memory task (same as Experiment 1). In addition, we were interested in comparing priming from the lexical decision task to priming from the word-familiarity task, and thus we employed a familiaritypriming task (same as Experiment 1). We reasoned that if the alternative explanation put forth above were true, then priming would be evident in the word-familiarly group and not in the lexical-decision group. If, however, this hypothesis is incorrect, then priming should be evident in both groups.

\section{Method}

\section{Participants}

The participants were 67 undergraduate students from Eastern Illinois University who participated in exchange for course credit. Twenty-two of the participants served in the lexicaldecision priming group and the control group, respectively, while 23 served in the word-familiarity priming group. Forty-three of the participants were females (16 in the lexical-decision group, 13 in the word-familiarity group, and 14 in the control group), and the age range of the participants was $18-20$ years ( $M=18.9$ years).

\section{Materials and procedure}

The lexical decision task contained 34 items that were printed and presented in booklet form. The list was similar to that used for the familiarity task, in that it contained all of the high- 
frequency items, with the difference that the low-frequency items were replaced with non-words (see Appendix 3). The non-word items were created to be pronounceable two- or three-syllable items (e.g., Relbar), having no reference in English or in any other language as legitimate words. The autobiographical memory task was the same task that was used in Experiment 1.

As in Experiments 1 and 2, priming group participants were first engaged in either the lexical decision task or the word familiarity task, followed by the autobiographical memory task, with time lags between tasks being around $5 \mathrm{~min}$. Each task was presented to participants as a separate study. Control group participants were only engaged in the autobiographical memory task. All participants were tested individually. Lexical decision task participants were instructed to read each item on the list and decide if items were words or not, indicating in the booklet "yes" in the affirmative and "no" in the negative, entering responses to the right of each word. The instructions for the word familiarity task were the same as in Experiments 1 and 2. All other instructions and procedures (i.e., for control group participants, for the autobiographical memory task) were that same as in Experiment 1.

\section{Categorization method}

Using the same criteria specified for Experiments 1 and 2, the judges examined the memories of the priming groups and the control group.

\section{Results and discussion}

An examination of the familiarity ratings in the word familiarity priming group showed that the overall ratings were 3.78 $(S D=0.23)$, while a breakdown of these ratings showed they were $4.92(S D=0.13)$ for high-frequency words, and 1.06 ( $S D$ $=0.61$ ) for low-frequency words. In the lexical decision group, all of the real words were correctly judged as words, while all of the non-words were correctly judged as such. Regarding the memory measures, we first analyzed performance on the wordcue task in terms of overall memory production, and general/ specific memory production. This analysis showed participants had very high rates of autobiographical memory generation on the word-cue task (96\% and $97 \%$, priming groups, $97 \%$, control group). Of these memories, $69-73 \%$ were specific autobiographical memories $(71 \%$ and $73 \%$ priming groups, $69 \%$ control group, $\chi^{2}(2)<1.0$ ), with the balances being general or summary autobiographical memories.

Turning to the main question of interest, an analysis of the conceptual overlap between items on the priming tasks and the autobiographical memory task showed that $.28(S D=.09)$ of the lexical decision group's memories were personal memories corresponding to items on their priming task, .26 ( $S D=$ .06 ) of the word familiarity group's memories had overlapped with their priming task, while the proportion was $.17(S D=$ $.06)$ for the control group (the Kappa statistic indicated high concordance between the judges, $K=.87, S E=.06$ ). An independent-samples analysis of variance revealed a significant different among the groups, $F(2,64)=14.71, M S E=$ $0.07, p<.001, \eta_{\mathrm{p}}{ }^{2}=.31$. Follow-up with Fischer's LSD statistic revealed that both priming groups significantly differed from the control group, while the priming groups did not differ from one another $(L S D=.04)$.

A further analysis of specific/general memories production rates in the overlap data showed specific memory rates similar to the overall data reported above (76\% and $77 \%$ priming groups, $73 \%$ control group, $\left.\chi^{2}(2)<1.0\right)$. An examination of the primed memories showed once again that all had corresponded to the high-frequency words, with 18 of the 23 high-frequency items being represented in the lexical decision group (78\%), many items occurring frequently; 17 of 23 in the word-familiarity group (74\%), many occurring frequently; 14 of 23 in the control group (61\%), occurring with less frequency.

Finally, at debriefing, participants indicated they had no knowledge of the research hypothesis, thus ruling out the possibility that the results were due to deliberate recall of memories associated with the items on the priming tasks.

In sum, showing priming in both the lexical decision and word familiarity groups argues against the alternative autobiographical-to-autobiographical priming hypothesis. As stated earlier, if this alternative hypothesis were true, then priming should have been evident in the word-familiarity priming group only. Instead, we observed priming in both the lexical decision and word familiarity groups. This result is best explained by the semantic-to-autobiographical priming hypothesis, as the autobiographical-to-autobiographical priming hypothesis is untenable in the case of the lexical-decision group. In order to advance the autobiographical-toautobiographical priming hypothesis for this group, one would have to claim that individuals need to retrieve personal memories matching the words in order to judge their lexical status. As such a claim appears to lack face validity, semantic-toautobiographical priming hypothesis remains the best explanation for the results observed in the two priming groups. Thus, the results of this experiment further support the semanticautobiographical priming hypothesis advanced in this study.

\section{General discussion}

The primary aim of this study was to show that the activation of semantic memories leads to the activation of autobiographical memories. The three experiments reported here each demonstrated support for this view. In Experiment 1, participants who were exposed to a word familiarity task showed more autobiographical memories corresponding to word familiarity items on a subsequent voluntary autobiographical memory 
task than control participants who had only received the latter task. In Experiment 2, participants who initially received the word familiarity task also showed more autobiographical memories related to word familiarity items than control participants; however, in this case the semantic-autobiographical priming effect was observed on a measure of involuntary autobiographical memories. Experiment 3 ruled out the alternative possibility that autobiographical memory processing had occurred in the word familiarity task by showing that semantic-autobiographical priming had occurred in a circumstance (lexical decision) where autobiographical memory processing is highly unlikely. Taken as a whole, the results support the idea that semantic-autobiographical memory activations are commonly occurring phenomena. Individually, the results of each experiment have implications for involuntary and voluntary recall of autobiographical memories.

At the broadest level, the results of this study have shown that the activation of concepts (e.g., summer, movie, music) results in the activation or priming of autobiographical memories involving such concepts (e.g., a personal memory about music). The data are consistent with results reported in Conway (1990), where concepts were shown to speed the retrieval of related autobiographical memories, and they support his general claim that autobiographical memories are continuously activated in the background by generic cognitive activity (e.g., see Conway, 2001). As can easily be imaged, for any significant segment of time (e.g., an hour or a day), this proposition suggests that generic processing would result in the activation of massive amounts of autobiographical memories. However, as argued at the outset, many of these activations never reach consciousness, some may immediately surface as involuntary autobiographical memories (Mace, 2010b), and as the data presented here argue, some are likely to surface later as voluntary (Experiments 1 and 3) or involuntary autobiographical memories (Experiment 2). Although our data may limit the conclusions that we can make, it seems that semantic-autobiographical priming would explain more everyday involuntary remembering than it would voluntary recall of the past, though the latter may depend on the circumstances in which voluntary recall is occurring.

In the case of voluntary recall, it seems unlikely that semantic-autobiographical priming would affect voluntary recall in the vast majority of everyday circumstances (e.g., when trying to remember what was discussed in a recent meeting, etc.), given the highly specific nature of this form of goaldirected voluntary recall and the relatively random nature of generic processing (e.g., what one may have read, or heard on television in a given day). Thus, it may be rare for these two elements to line up such that prior semantic processing would be in a position to either facilitate or inhibit the voluntary recall of autobiographical memories (e.g., having processed information that would increase the likelihood of recalling what was discussed at that recent meeting). However, given the relatively unstructured nature of reminiscing (e.g., freely recalling when one was a child), semantic-autobiographical priming probably plays a larger role in this form of voluntary recall.

Our data seem to support this idea. Autobiographical memories related to priming task concepts were observed in a retrieval environment that bears some similarity to reminiscing. That is, while participants were required to produce memories related to the cue words, they were free to choose among a set of possible different memories that may be deemed as directly related to the cue words (e.g., for the cue word garden, one could have a large set of memories). We believe that this is like some forms of everyday reminiscing, as the goals of the task can be seen as relatively broad (e.g., in reminiscing about the past, one is free to choose from a large set of memories). Semanticautobiographical priming in these circumstances may narrow (or direct) the field of potential memories, such that memories that share associated features have an increased likelihood of retrieval. So, for example, in the case of the autobiographical memory word-task, the cue word garden may make a memory about gardening in the summer (where summer is the primed concept) more likely to be retrieved than a memory about buying seeds for the garden (an unprimed case). In the case of naturally occurring reminiscing, one would be more prone to recall memories about summer when reminiscing about childhood than other unprimed concepts. Thus, semantic-autobiographical priming may have little impact on the typical form of goal-direct voluntary recall, while its role in reminiscence-type voluntary recall could be substantially greater.

In comparison to voluntary autobiographical memories, involuntary autobiographical memories typically do not occur in goal-directed circumstances (for exceptions, see Mace, 2006, 2007a), but occur spontaneously throughout the course of the day. The spontaneous nature of involuntary memories should substantially increase the possibility that the two processes (semantic-autobiographical priming and involuntary autobiographical remembering) will line up with one another. For example, in the case where summer is the conceptual prime, spontaneous memories about summer could be retrieved when one subsequently encounters directly related cues (e.g., hearing or seeing the word summer in another context) or indirectly related cues (e.g., hearing someone say the word gardening triggers a memory about gardening in the summer). It seems that the possibilities and opportunities here are massive, and as indicated at the outset, semanticautobiographical priming could potentially account for a great deal of everyday involuntary autobiographical remembering. For example, a number of studies and writers have suggested that a portion of involuntary memories may be functional (i.e., serve some purpose), whereas another portion of them may be non-functional or random (see, Berntsen, 2009; Mace, 2017; Mace \& Atkinson, 2009; Rasmussen \& Berntsen, 2009; Rasmussen et al., 2015). Perhaps semantic-autobiographical 
priming accounts for a good proportion of the random component of involuntary memories.

One question that arises from the above formulations is: are the voluntary and involuntary autobiographical memories that result from semantic-autobiographical memory priming functional or merely a consequence of the priming process? In the present study we have considered autobiographical memories that arise outside of the original activation or priming event. In other words, autobiographical memories that may occur minutes to hours later in some other processing context. It would seem that these memories, as opposed to ones that may occur immediately in the priming context, are likely to be a nonfunctional consequence of priming, as they are now likely to be task-irrelevant. Consequently, memories that occur in deliberate reminiscing or involuntary memories that occur days, hours, or even minutes after the priming event are not likely to be functional to their current processing context. Thus, we believe that while semantic-autobiographical memory priming may explain a good portion of everyday involuntary remembering, for example, it also might support that idea that much of involuntary remembering is not functional to everyday cognition, as these memories are merely a consequence of semanticautobiographical priming (for discussions on the possible nonfunctional nature of involuntary memories, see Mace, 2018; Mace \& Atkinson, 2009; and Rasmussen et al., 2015).

Interestingly, however, showing that semanticautobiographical priming results in the production of involuntary autobiographical memories can lead one to the conclusion that such involuntary memories are a part of an orderly cognitive process. That is, if semantic-autobiographical priming is responsible for some everyday involuntary memory production, as the data presented here would suggest, then viewed in isolation, these memories should not be seen as merely random cognitive events, as they would be the result of prior cognitive activity. This idea adds to the general argument that involuntary autobiographical memories are better understood as set of organized cognitive events that tracks recent thoughts and reminiscences, rather than purely random slips of cognition (see further discussions in Barzykowski \& Niedzwienska, 2017, and Mace, 2005).

Although we have suggested that semantic-autobiographical priming may last for hours or days, the priming observed in this study occurred after a time lag of just several minutes, indicating that the time course of semantic-autobiographical priming is at least several minutes, or lasting no longer than this period. While we cannot conclude from our data that semantic-autobiographical priming lasts longer than several minutes, there is good reason to believe that it lasts for hours to days. In the Mace (2005) study, autobiographical memory priming effects were observed days after the priming sessions. Given these results, the autobiographical memory component of semantic-autobiographical priming should last for hours to days, even if the semantic memory component fades within minutes, as suggested by traditional semantic priming studies (see Anderson, 1983a). However, more recent naturistic observations of semantic priming have shown that semantic priming can last for periods considerably longer than the seconds or minutes suggested by more traditional semantic priming studies (i.e., see details and review in Coane \& Balota, 2009; Kvavilashvili \& Mandler, 2004, where priming occurred after days or longer). Thus, we believe that it is highly likely that semantic-autobiographical priming may endure for hours to days. If this is true, then its effects on everyday autobiographical memory production may indeed be quite substantial, as it would indicate a massive reserve of both recent and more remote activations in the autobiographical memory system.

However, it should be noted that there are probably factors that modulate the type and amount of personal memories that are primed by semantic memories. One likely factor concerns a concept's association with personal experience. That is, it seems that concepts naturally range from those that are weakly associated with autobiographical memories to those that are strongly associated with autobiographical memories. Weakly associated concepts may activate fewer autobiographical memories in general, and when they do, they are probably more likely to activate general autobiographical memories rather than specific autobiographical memories (i.e., episodic memories). On the other hand, concepts that are more strongly associated with autobiographical memories should activate more autobiographical memories overall (i.e., both general and specific forms). Individual experience is also likely to be a moderating factor. For example, the concept stop sign may be associated with very few autobiographical memories for most individuals, but this may not be the case for traffic cops.

In explaining why taxonomic categories failed to prime autobiographical memories, while goal-derived categories did, Conway (1990) offered a somewhat similar type of association strength view (see details in his study). However, we believe that the data presented here shed a somewhat different light on, and perhaps interpretation of, Conway's findings. First, it is instructive to note that while no priming was obtained with taxonomic categories in that study, taxonomic categories did consistently show shorter retrieval latencies in comparison to control conditions, thus suggesting that they may fit into a weak association category. Second, it is also instructive to note that many of the items used on our priming tasks were taxonomic categories (sports, music, car, clothing) or exemplars of taxonomic categories (e.g., home, books, cat, see Battig \& Montague, 1969), and they were strongly represented in our priming data (i.e., appearing frequently as memory topics). Thus, we believe that our results show that an association strength continuum (weak vs. strong) does not necessarily line up with a taxonomic versus goal-derived category continuum, but more simply varies in accord with a concept's association with personal experience. Further, we believe that our results show that our measure of priming (direct 
observations of content) is more sensitive to semanticautobiographical priming overall than retrieval latency measures, as we obtained priming results where Conway did not.

An association strength argument can also explain why priming in this study was prevalent among common (or high familiarity) concepts (e.g., summer, hiking, movie), but nonexistent among uncommon (or low familiarity) concepts (e.g., garrulous, pensive, stop sign). Indeed, many of the items that we used in the uncommon category were probably so weakly associated with autobiographical memories that they would not register on typical measures of autobiographical memory. For example, imagine that the item garrulous is incapable of capturing any episodic or general autobiographical memories, only fragmentary aspects of the two (e.g., a face or a name), which would not register on any standard measure of autobiographical memory. Similarly, in the case of the item stop sign, unless our participants were traffic cops or frequent violators of traffic laws, this concept might only prime a personal image of a stop sign (e.g., an image of one on the corner where one lives), not a fully blown episodic memory involving one.

At a broader level, the results of this study also have implications for semantic priming theory. In classical semantic priming theory, spreading activation is seen as functional because the spread of activation occurs amongst associated information that is potentially relevant to the current processing context (for a review, see Anderson, 1983a; and classic studies on spreading activation, e.g., Anderson, 1983b; Collins \& Loftus, 1975, as well as alternative compound cue theories, e.g., Ratcliff \& McKoon, 1988; and rebuttals in McNamara, 1992a, b, 1994). Thus, memories that are activated or retrieved within a particular processing context are potentially functional to that context. Showing here that the spread of activation also extends to related autobiographical memories suggests that they, too, are potentially relevant to the processing context. We believe that this proposition adds a new dimension to theories of semantic priming, as they will need to incorporate the notion that general knowledge structures activate related general knowledge structures as well as personal knowledge structures (or autobiographical memories). Theories directed toward such a view will also need to address how (and why) personal knowledge structures are functionally relevant to processing contexts (e.g., sentence or story comprehension), including the possibility that they may not always be functional (i.e., in cases where autobiographical knowledge activation distracts from processing).

Thus from a broader perspective, the data presented here strongly suggest that semantic access also involves autobiographical knowledge access. The findings, therefore, have implications for how we process the world, and as suggested directly by our data, implications for linguistic processing. At a functional level, the results suggest that lexical access also involves autobiographical memory access, and perhaps this occurs because personal experience is relevant in the normal course of language processing. Our understanding of language may not be limited to the access of general concepts, but also may be dependent on the access of relevant personal experiences. Such an idea has implications for theories of semantic memory, and the best fit for such an approach might be grounded theories of cognition and semantic memory (e.g., Barsalou, 1999; Patterson, Nestor, \& Rogers, 2007).

The idea that personal experiences (or autobiographical memories) may be integral to how we understand the world and language also has implications for memory systems theories (e.g., Schacter \& Tulving, 1994; Squire, 2004; Tulving, 1972), particularly the relationship between semantic memory and autobiographical memory. Traditionally considered as independently functioning systems, with the exception that episodic memory (or autobiographical memory) had some reliance on semantic memory (e.g., Tulving, 1972, 1983, 2002), our data, and the ideas being advanced here, suggest that these two systems may be more interdependent (or interactive) than originally conceived (see also, Conway, 1990, 2005). Several processes might mediate their interaction, and Barsalou's (1999) and Conway's (2005) approaches to semantic and autobiographical memory, respectively, may be instructive here.

For example, when semantic access occurs, associated generic images (e.g., the image symbol for a $d o g$ ), and other perceptual features, may be activated (Barsalou, 1999). These activations may in turn capture related episodic (experientially based) images (e.g., my $d o g$ ), resulting in related specific autobiographical memory activations (episodic memories). A more top-down (or abstract) process may occur when semantic activations (e.g., the word department) cause activations of related autobiographical knowledge at the general-event layer of autobiographical memory (e.g., my department), which in turn activate sets of associated episodic memories (Conway, 2005).

In conclusion, the results of this study have firmly established that semantic memory activation results in autobiographical memory activation. We have argued that the results support the notion that continuous semantic activation results in continuous autobiographical memory activation, the products of which are likely to impact everyday involuntary and voluntary autobiographical memory production. While the goals and the scope of this study were limited to these issues, the results also have a set of broader implications, which as is evident by the foregoing discussion, open up a wealth of questions for future research. In particular, these involve determining the time course of semanticautobiographical priming, determining the nature and range of semantic-autobiographical memory associations, as well as examining how semantic-autobiographical priming may modify aspects of semantic priming theory, thus leading to questions on the functional nature of autobiographical knowledge access in everyday cognition. Last, but not least, are the questions regarding the interdependence of semantic memory and autobiographical memory. 
Appendix 1 Words used in the word familiarity task

\begin{tabular}{ll}
\hline Music* & Clothing* \\
Friends* & Books* \\
Schoö* & Garrulous** \\
Quip** & Cell phone* \\
Graduation* & City* \\
Parade* & Lilt** \\
Art* & Internet* \\
Pensive** & Cat* \\
Doctor* & Haphazard** \\
Vacation* & Cartoons* \\
Home* & Reading* \\
Ostentatious** & Proclivity** \\
Pet* & Hiking* \\
Summer* & Movie* \\
Blatant** & Sports* \\
Stop Sign** & Asunder** \\
Scruple** & Car* \\
\hline
\end{tabular}

*Denotes a high-frequency word

$* *$ Denotes a low-frequency word
Appendix 3 Words used in the lexical decision task

Music*

Friends*

Clothing*

School*

Manac**

Graduation*

Parade*

Art*

Fortan**

Books*

Relbar**

Cell phone*

City*

Lilorit**

Doctor*

Internet*

Cat*

Vacation*

Pardhal**

Home*

Cartoons*

Reading*

Denma**

Prosum**

Pet*

Hiking*

Summer*

Movie*

Turcul**

Sports*

Monder**

Suntle**

Pulel**

*Denotes a high-frequency word

**Denotes a non-word

\section{References}

\section{Appendix 2 C
memory task}

Flower

Mountain

Train

Restaurant

Baby

Party

Window

Cup

Market

Chair

Bicycle

Park

Leaves

Family

Pool

Anderson, J.R. (1983a). The architecture of cognition. Cambridge MA: Harvard University Press.

Anderson, J. R. (1983b). A spreading activation theory of memory. Journal of Verbal Learning and Verbal Behavior, 22, 261-295.

Ball, C.T. (2007). Can we elicit involuntary autobiographical memories in the laboratory? In J.H. Mace (Ed.), Involuntary memory (pp. 127152). Malden, MA: Blackwell Publishing.

Ball, C.T., \& Hennessey, J. (2009). Subliminal priming of autobiographical memories. Memory, 17, 311-322.

Ball, C. T., \& Little, J. C. (2006). A comparison of involuntary autobiographical memory retrievals. Applied Cognitive Psychology, 20, 1167-1179.

Ball, C. T., Mace, J. H., \& Corona, H. (2007). Cues to the gusts of memory. In J. H. Mace (Ed.), Involuntary memory (pp. 113-126). Malden, MA: Blackwell Publishing.

Barsalou, L.W. (1988). The content and organization of autobiographical memories. In U. Neisser, \& E. Winograd (Eds.), Remembering reconsidered: Ecological and traditional approaches to the study of memory (pp. 193-243). New York: Cambridge University Press.

Barsalou, L.W. (1999). Perceptual symbols systems. Behavioral and Brain Sciences, 22, 577-660.

Barzykowski, K., \& Niedzwienska, A. (2017). Priming involuntary autobiographical memories in the lab. Memory.

Barzykowski, K., \& Staugaard, S.R., (2016). Does retrieval intentionality really matter? Similarities and differences between involuntary memories and directly and generatively retrieved voluntary memories. British Journal of Psychology, 107, 519-536.

Battig, W.F., \& Montague, W.E. (1969). Category norms for verbal items in 56 categories. Journal of Experimental Psychology, 80, 1-46.

Berntsen, D. (1996). Involuntary autobiographical memory. Applied Cognitive Psychology, 10, 435-454. 
Berntsen, D. (1998). Voluntary and involuntary access to autobiographical memory. Memory, 6, 113-141.

Berntsen, D. (2009). Involuntary autobiographical memories: An introduction to the unbidden past. Cambridge: Cambridge University Press.

Coane, J.H., \& Balota, D.A. (2009). Priming the holiday spirit: Persistent activation due to extra-experimental experiences. Psychonomic Bulletin \& Review, 16, 1124-1128.

Collins, A. M., \& Loftus, E. F. (1975). A spreading-activation theory of semantic processing. Psychological Review, 82, 407-428.

Connine, C.M., Mullennix, J., Shernoff, E., \& Yelen, J. (1990). Word familiarity and frequency in visual and auditory word recognition. Journal of Experimental Psychology: Learning, Memory, and Cognition, 16, 1084-1096.

Conway, M. A. (1990). Associations between autobiographical memories and concepts. Journal of Experimental Psychology: Learning, Memory, and Cognition, 16, 799-812.

Conway, M.A. (2001). Sensory-perceptual episodic memory and its context: Autobiographical memory. In A. Baddeley, J.P. Aggleton, \& M.A. Conway (Eds.), Episodic memory: New directions in research (pp. 53-70). New York: Oxford University Press.

Conway, M. A. (2005). Memory and the self. Journal of Memory and Language, 53, 594-628.

Conway, M.A., \& Bekerian, D.A. (1987). Organization in autobiographical memory. Memory \& Cognition, 15, 119-132.

Fitzgerald, J.M. (1996). Intersecting meanings of reminiscence in adult development and ageing. In D.C. Rubin (Ed.), Remembering our past: Studies in autobiographical memory (pp. 360-383). New York: Cambridge University Press.

Kvavilashvili, L., \& Mandler, G. (2004). Out of one's mind: A study of involuntary semantic memories. Cognitive Psychology, 48, 47-94.

Mace, J.H. (2004). Involuntary autobiographical memories are highly dependent on abstract cuing: The Proustian view is incorrect. Applied Cognitive Psychology, 18, 893-899.

Mace, J.H. (2005). Priming involuntary autobiographical memories. Memory, 13, 874-884.

Mace, J.H. (2006). Episodic remembering creates access to involuntary conscious memory: Demonstrating involuntary recall on a voluntary recall task. Memory 14, 917-924.

Mace, J.H. (2007a). Does involuntary remembering occur during voluntary remembering? In J.H. Mace (Ed.), Involuntary memory (pp. 5067). Malden, MA: Blackwell Publishing.

Mace, J.H. (Ed.) (2007b). Involuntary memory. Malden, MA: Blackwell Publishing.

Mace, J.H. (2010a). Involuntary remembering and voluntary remembering: How different are they? In J.H. Mace (Ed.), The act of remembering: Toward an understanding of how we recall the past (pp. 183-201). Malden, MA: Wiley-Blackwell.

Mace, J.H. (2010b). Understanding autobiographical remembering from a spreading activation perspective. In J.H. Mace (Ed.), The act of remembering: Toward an understanding of how we recall the past (pp. 43-55). Malden, MA: Wiley-Blackwell.

Mace, J.H., (2018). Involuntary autobiographical memories: Spontaneous recollections of the past. In K.C.R. Fox, \& K. Christoff (Eds.), The Oxford handbook of spontaneous thought: Mind-wandering, dreaming, creativity, and clinical disorders, Oxford: Oxford University Press.

Mace, J.H., \& Atkinson, E. (2009). Can we determine the functions of everyday involuntary autobiographical memories? In M. Kelly (Ed.), Applied memory, New York: Nova Science Publishers.

Mace, J.H., \& Clevinger, A.M. (2013). Priming voluntary autobiographical memories: Implications for the organization of autobiographical memory and voluntary recall processes. Memory, 21, 524-536.
Mace, J.H., Clevinger, A.M., \& Bernas, R. S. (2013). Involuntary memory chains: What do they tell us about autobiographical memory organization? Memory, 21, 324-335.

McNamara, T. P. (1992a). Priming and constraints it places on theories of memory and retrieval. Psychological Review, 99, 650-662.

McNamara, T. P. (1992b). Theories of priming: I. Associative distance and lag. Journal of Experimental Psychology: Learning, Memory, and Cognition, 18, 1173-1190.

McNamara, T. P. (1994). Theories of priming: II. Types of primes. Journal of Experimental Psychology: Learning, Memory, and Cognition, 20, 507-520.

Patterson, K.E., Nestor, P.J., \& Rogers, T.T. (2007). Where do you know what you know? The representation of semantic knowledge in the human brain. Nature Reviews Neuroscience, 8, 976-987.

Rasmussen A.S., \& Berntsen, D. (2009). The possible functions of involuntary autobiographical memories. Applied Cognitive Psychology, 23, 1137-1152.

Rasmussen A.S., \& Berntsen, D. (2011). The unpredictable past: Spontaneously retrieved autobiographical memories outnumber autobiographical memories retrieved strategically. Consciousness and Cognition, 20, 1842-1846.

Rasmussen, A.S., Ramsgaard, \& Berntsen, D. (2015). The frequency and functions of involuntary and voluntary autobiographical memories across the day. The Psychology of Consciousness: Theory, Research and Practice, 2, 185-205.

Ratcliff, R., \& McKoon, G. (1988). A retrieval theory of priming in memory. Psychological Review, 95, 385-408.

Roediger, H.L., III, \& McDermott, K. (1993). Implicit memory in normal human participants. In F. Boller \& J. Grafman (Eds.), Handbook of neuropsychology (Vol. 8, pp. 63-131). New York: Elsevier Science.

Rubin, D.C., Wetzler, S.E., \& Nebes, R.D. (1986). Autobiographical memory across the lifespan. In D.C. Rubin (Ed.), Autobiographical memory (pp. 202-221). New York: Cambridge University Press.

Schacter, D.L. (1992). Priming and multiple memory systems: Perceptual mechanisms of implicit memory. Journal of Cognitive Neuroscience, 4, 244-256.

Schacter, D. L., \& Tulving, E. (1994). What are the memory systems of 1994? In D. L. Schacter \& E. Tulving (Eds.), Memory systems 1994 (pp. 1-38). Cambridge, MA: MIT Press.

Schlagman, S., \& Kvavilashvili, L. (2008). Involuntary autobiographical memories in and outside the laboratory: How different are they from voluntary autobiographical memories? Memory \& Cognition, 36, 920-932.

Schlagman, S., Kvavilashvili, L., \& Schulz, J. (2007). Effects of age on involuntary autobiographical memories. In J.H. Mace (Ed.), Involuntary memory (pp. 87-112). Malden, MA: Blackwell Publishing.

Squire, L. R. (2004). Memory systems of the brain: A brief history and current perspective. Neurobiology of Learning and Memory, 82, $171-177$

Tulving, E. (1972). Episodic and semantic memory. In E. Tulving \& W. Donaldson (Eds.), Organization of memory (pp. 381-402). New York, NY: Academic Press.

Tulving, E. (1983). Elements of episodic memory. Oxford: Clarendon.

Tulving E. (2002). Episodic memory: From mind to brain. Annual Review of Psychology, 53, 1-25.

Vannucci, M., Pelagatti, C., Hanczakowski, M., Mazzoni, G., \& Paccani, C.R. (2015). Why are we not flooded by involuntary autobiographical memories? Few cues are more effective than many. Psychological Research, 79, 1077-1085. 